\title{
Further consideration of closure at the root of a sharp notch
}

H Qiu*, D A Hills, and D Dini

Department of Engineering Science, University of Oxford, Oxford, UK

The manuscript was received on 14 January 2008 and was accepted after revision for publication on 11 March 2008.

DOI: 10.1243/03093247JSA404

Abstract: The true contact length and corresponding contact pressure distribution arising at the root of a semi-infinite sharp notch, subject to compression, is found.

Keywords: closure, compressive force, sharp notch, contact length, contact pressure

\section{INTRODUCTION}

The problem to be addressed in this paper is the quantification of the closure region when a notch having a sharp root is subjected to a compressive force, tending to close it. This is of practical importance in estimating the fatigue life of a sharp notch, and the possibility of nucleating a crack, because the local state of stress which the nucleating crack experiences will depend on the degree of closure, and whether or not it encompasses the 'process zone'. It is not necessary to examine the problem of a finite notch within whatever geometry it exists, because the closure zone will, for practical purposes, nearly always be small compared with the crack length. It is therefore appropriate to consider the behaviour of a semi-infinite notch, and then to use the generalized stress intensity factor, scaling the notch solution to fit it into whatever finite problem is being examined. Two of the present authors and a co-worker [1] have already looked at this problem once, using merely the Williams notch solution itself, and checking for violations in the surface displacements, i.e. finding the point at which the surface displacements of the notch faces suggest that there will be interpenetration. This is clearly a useful first step in estimating the amount of closure, but it is approximate and will be rather poor when the external notch half-angle $\alpha$ (Fig. 1) approaches $\pi \mathrm{rad}$ or becomes crack like. In order to solve the problem the general method of attack will be initially to permit interpenetration, as indicated by the dashed curves in Fig. 1(a), and then to apply a distributed contact pressure along the notch flanks so as to push the surfaces back to a common boundary. This solution has become feasible because the influence function for a pair of equal opposed forces applied to the surfaces of a notch has been solved [2], and this will form the kernel of an integral equation formulation for this problem.

\section{FORMULATION}

The first step is to deduce the form of the surface normal displacement of the notch itself, due to a remote applied load. Note that, in general, a remote load might be expected to induce both a mode I (symmetric) field and a mode II (antisymmetric) field, but the latter will cause only a local rotation of the notch and will not affect the separation of the notch faces. The surface normal displacement may be found directly from the Williams [3] asymptotic form and is given by

$$
2 \mu u_{\theta}^{\mathrm{I}}(r, \theta)=-K_{\mathrm{I}}^{\mathrm{S}} r^{\lambda_{\mathrm{I}}} \frac{\left(\lambda_{\mathrm{I}}-1\right)\left\{\sin \left[\left(\lambda_{\mathrm{I}}-1\right) \alpha\right] / \sin \left[\left(\lambda_{\mathrm{I}}+1\right) \alpha\right]\right\} \sin \left[\left(\lambda_{\mathrm{I}}+1\right) \theta\right]-\left(\kappa+\lambda_{\mathrm{I}}\right) \sin \left[\left(\lambda_{\mathrm{I}}-1\right) \theta\right]}{\lambda_{\mathrm{I}}\left(\lambda_{\mathrm{I}}+1\right)\left\{1-\cos \left[\left(\lambda_{\mathrm{I}}-1\right) \alpha\right] / \cos \left[\left(\lambda_{\mathrm{I}}+1\right) \alpha\right]\right\}}, \quad-\alpha \leqslant \theta \leqslant \alpha
$$

*Corresponding author: Department of Engineering Science, University of Oxford, Parks Road, Oxford OX1 3PJ, UK. email: hui.qiu@eng.ox.ac.uk 
(a)

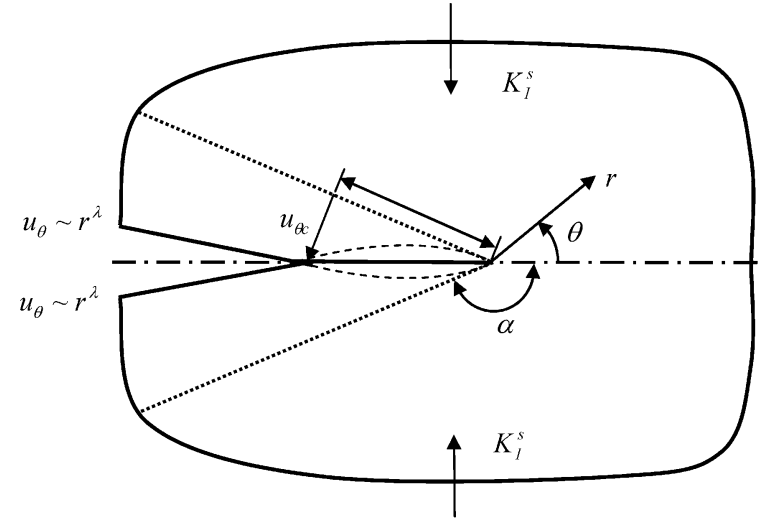

(b)

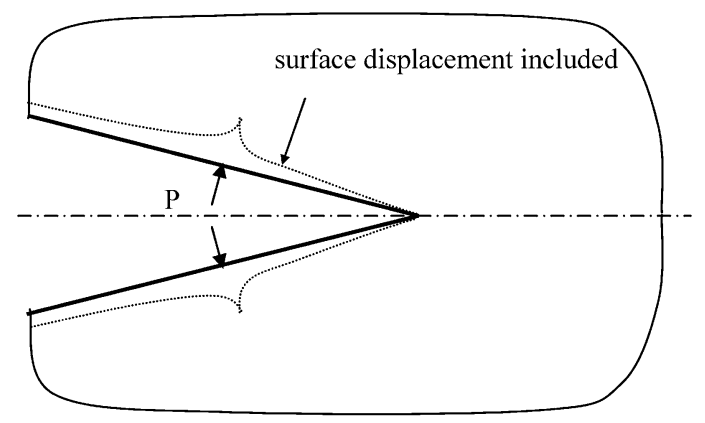

Fig. 1 (a) 'Overlap' and normal displacements along the notch flanks; (b) a point force applied normal to the notch faces

where the generalized stress intensity factor $K_{\mathrm{I}}^{\mathrm{s}}$ is given by

$$
K_{\mathrm{I}}^{\mathrm{S}}=\lim _{r \rightarrow 0} \frac{\sigma_{\theta \theta}(r, 0)}{r^{\lambda_{\mathrm{I}}-1}}
$$

It follows that the relative normal displacement between the two notch surfaces may be found by specializing equation (1), by setting $\theta= \pm \alpha$, to give

$$
u_{\theta}^{\mathrm{I}}(r, \alpha)=\frac{K_{\mathrm{I}}^{\mathrm{s}} r^{\lambda_{\mathrm{I}}}}{E^{*}} \Lambda
$$

where

$$
\Lambda=\frac{\sin (2 \alpha)}{\lambda_{\mathrm{I}} \sin \alpha \sin \left(\lambda_{\mathrm{I}} \alpha\right)}=\frac{2 \cos \alpha}{\lambda_{\mathrm{I}} \sin \left(\lambda_{\mathrm{I}} \alpha\right)}
$$

In order to establish the true closure length $r_{\mathrm{c}}$ an integral equation in terms of the contact pressure will be set up. This could be expressed in terms of the surface displacement itself, but, in practice, it is preferable to work in terms of the surface displacement gradient $\partial u_{\theta}^{\mathrm{I}} / \partial r$, as this means that the kernel will be Cauchy rather than logarithmic in nature and hence become easier to handle. With this in mind, the boundary condition along the closure line is written as

$$
\frac{\partial u_{\theta}^{\mathrm{I}}(r, \alpha)}{\partial r}-\frac{\partial u_{\theta}^{\mathrm{p}}(r, \alpha)}{\partial r}=\tan (\pi-\alpha)
$$

where $u_{\theta}^{\mathrm{p}}(r, \alpha)$ is the displacement due to a continuous distribution of line forces along the edge contact and is given by

$$
\frac{\partial u_{\theta}^{\mathrm{p}}(r, \alpha)}{\partial r}=\int_{0}^{r_{\mathrm{c}}} p(\xi) * D_{\theta}^{\mathrm{m}}[r, \alpha, \xi] \mathrm{d} \xi, \quad 0 \leqslant r \leqslant r_{\mathrm{c}}
$$

Here $D_{\theta}^{\mathrm{m}}[r, \alpha, \xi]$ is the displacement derivative influence function for opposed line forces applied to a semi-infinite wedge [2]. By setting

$$
s=\frac{2 \xi}{r_{\mathrm{c}}}-1
$$

and

$$
t=\frac{2 r}{r_{\mathrm{c}}}-1
$$

the normalized interval of the integral equation becomes

$$
\begin{aligned}
\frac{\partial u_{\theta}^{\mathrm{I}}(t, \alpha)}{\partial r}-\tan (\pi-\alpha)= & \frac{r_{\mathrm{c}}}{2} \int_{-1}^{1} p(s) * D_{\theta}^{\mathrm{m}}[t, \alpha, s] \mathrm{d} s \\
& -1 \leqslant s \leqslant 1
\end{aligned}
$$

It is known that, at the corner of the notch, the stress is square root singular and at the contact edge, it is bounded. In fact, two arguments point to the fact that the local behaviour is square root bounded in character at the edge. First, from basic RiemannHilbert theory, it is known that a singular integral equation with a Cauchy kernel must display local behaviour which is either square root singular or square root bounded. Here, the kernel has a generalized Cauchy character, and it is assumed that the same characteristic will apply. The second argument is that, very close to the edge of the contact, halfplane theory may be applied and, if the profile gradient of the indenting surfaces is represented by a Taylor series, the first term corresponds to a local square root contact pressure distribution. Therefore it can be stated that

$$
p(s)=p_{\alpha}(s)(1-s)^{1 / 2}(1+s)^{-1 / 2}
$$


The discretized form of the integral equation is

$$
\begin{gathered}
\frac{\partial u_{\theta}^{\mathrm{I}}\left(t_{k}, \alpha\right)}{\partial r}-\tan (\pi-\alpha)=\frac{r_{\mathrm{c}}}{2} \sum_{i=1}^{n} W_{i} p_{\alpha}\left(s_{i}\right) D_{\theta}^{\mathrm{m}}\left[t_{k}, \alpha, s_{i}\right] \\
k=1, \ldots, n
\end{gathered}
$$

where

$$
\begin{aligned}
& s_{i}=\cos \left(\frac{2 i}{2 n+1} \pi\right), \quad i=1, \ldots, n \\
& t_{k}=\cos \left(\frac{2 k-1}{2 n+1} \pi\right), \quad k=1, \ldots, n \\
& W_{i}=\frac{2 \pi\left(1-s_{i}\right)}{2 n+1}
\end{aligned}
$$

These represent a system of $n$ simultaneous equations for $n$ unknown $p_{\alpha}\left(s_{i}\right)$, together with the closure length $r_{\mathrm{c}}$.

A first estimate of the closure distance, $r_{\mathrm{c}}^{\mathrm{o}}$, is found from the point of overlap of the Williams solution and is given by

$$
\begin{aligned}
r_{\mathrm{c}}^{\mathrm{o}} & =\left[\frac{K_{\mathrm{I}}^{\mathrm{s}}}{E^{*}} \frac{\Lambda}{\tan (\pi-\alpha)}\right]^{1 /\left(1-\lambda_{\mathrm{I}}\right)} \\
& =\left[\frac{K_{\mathrm{I}}^{\mathrm{s}}}{\mu} \frac{(1-v) \cos \alpha}{\lambda_{\mathrm{I}} \sin \left(\lambda_{\mathrm{I}} \alpha\right) \tan (\pi-\alpha)}\right]^{1 /\left(1-\lambda_{\mathrm{I}}\right)}
\end{aligned}
$$

The true closure length $r_{\mathrm{c}}$ is found iteratively; the contact pressure must be a negative value along the contact interface, and the notch must be open external to the contact distance. Improved values of $r_{\mathrm{c}}$ are chosen until their two inequalities are satisfied.

Because of the limitation of linear elastic theory, which explicitly excludes rotation effects, the solution will apply only if $2 \alpha$ is large. Notches of $300^{\circ}$, $330^{\circ}$, and $350^{\circ}$ were chosen as detailed examples. The influence functions for these cases are as follows. For a $350^{\circ}$ notch,

$$
\begin{aligned}
D_{\theta}^{\mathrm{m}}[r, \alpha, \xi] & =\frac{1}{\mu}\left[-\frac{4.628 r^{2}}{(1+\sqrt{r / \xi})^{5} \xi^{3}}\right. \\
& +\frac{8.0655 r^{2}}{(1+\sqrt{r / \xi})^{4} \sqrt{r / \xi} \xi^{3}}-\frac{15.606 r}{(1+\sqrt{r / \xi})^{3} \xi^{2}} \\
& +\frac{0.8205 r}{(1+\sqrt{r / \xi})^{2} \sqrt{r / \xi} \xi^{2}}-\frac{1.641}{(1+\sqrt{r / \xi}) \xi} \\
& -\frac{0.0805}{\sqrt{r / \xi} \xi}+\frac{7.278 \sqrt{r / \xi}}{(1+\sqrt{r / \xi})^{2} \xi} \\
& \left.+\frac{5.785(r / \xi)^{3 / 2}}{(1+\sqrt{r / \xi})^{4} \xi}+\frac{1-v}{\pi \xi} \frac{1}{r / \xi-1}\right]
\end{aligned}
$$

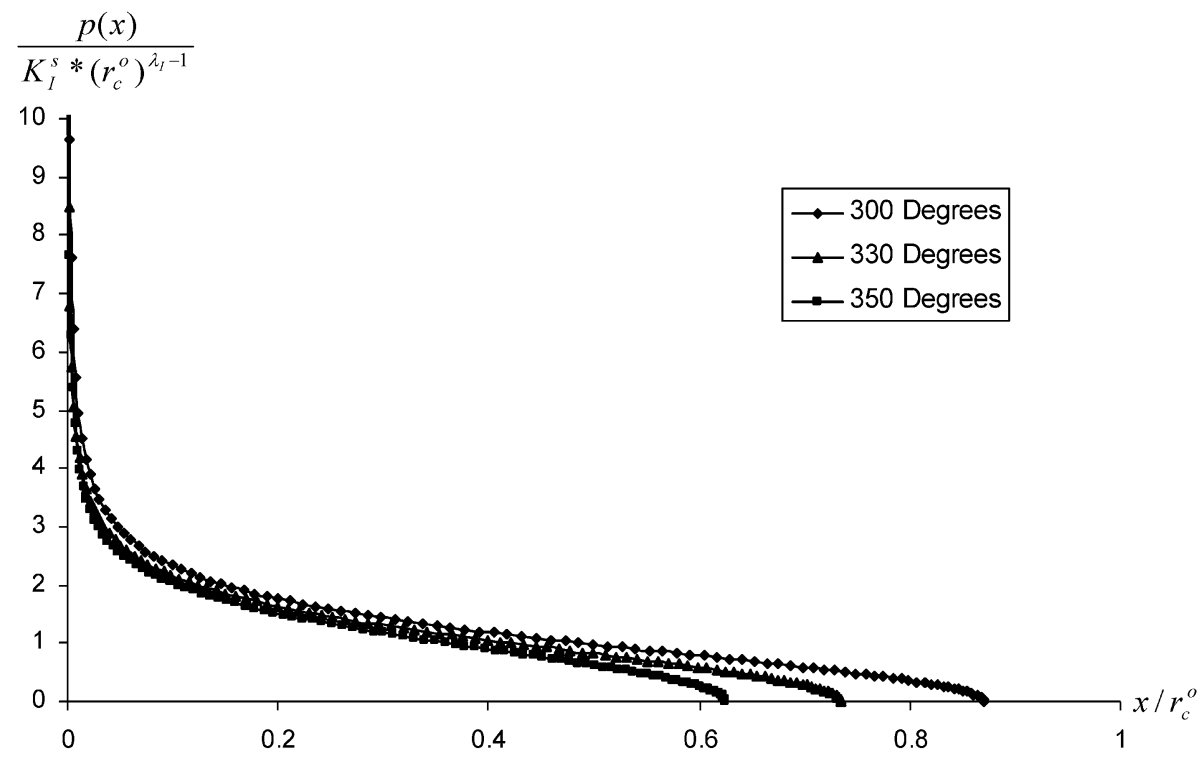

Fig. 2 Predicted interfacial contact pressure and true closure length 
Table 1 Values of $\lambda_{\mathrm{I}}$ and $C$ for the three cases studied $\left(350^{\circ}, 330^{\circ}\right.$, and $300^{\circ}$ notches $)$, together with those for a $340^{\circ}$ notch

\begin{tabular}{lll}
\hline Notch angle & $\lambda_{\mathrm{I}}$ & $C$ \\
\hline $350^{\circ}$ & 0.50005 & 1.692 \\
$340^{\circ}$ & 0.50043 & 1.559 \\
$330^{\circ}$ & 0.50145 & 1.553 \\
$300^{\circ}$ & 0.51222 & 1.362 \\
\hline
\end{tabular}

For a $330^{\circ}$ notch,

$$
\begin{aligned}
D_{\theta}^{\mathrm{m}}[r, \alpha, \xi] & =\frac{1}{\mu}\left[-\frac{2.946 r^{2}}{(1+\sqrt{r / \xi})^{5} \xi^{3}}\right. \\
& +\frac{5.0235 r^{2}}{(1+\sqrt{r / \xi})^{4} \sqrt{r / \xi} \xi^{3}}-\frac{9.754 r}{(1+\sqrt{r / \xi})^{3} \xi^{2}} \\
& +\frac{0.4855 r}{(1+\sqrt{r / \xi})^{2} \sqrt{r / \xi} \xi^{2}}-\frac{0.971}{(1+\sqrt{r / \xi}) \xi} \\
& -\frac{0.106}{\sqrt{r / \xi}+\frac{4.584 \sqrt{r / \xi}}{(1+\sqrt{r / \xi})^{2} \xi}} \\
& \left.+\frac{3.6825(r / \xi)^{3 / 2}}{(1+\sqrt{r / \xi})^{4} \xi}+\frac{1-v}{\pi \xi} \frac{1}{r / \xi-1}\right]
\end{aligned}
$$

For a $300^{\circ}$ notch,

$$
\begin{aligned}
D_{\theta}^{\mathrm{m}}[r, \alpha, \xi] & =\frac{1}{\mu}\left[-\frac{2.864 r^{2}}{(1+\sqrt{r / \xi})^{5} \xi^{3}}\right. \\
& +\frac{5.0505 r^{2}}{(1+\sqrt{r / \xi})^{4} \sqrt{r / \xi} \xi^{3}}-\frac{9.773 r}{(1+\sqrt{r / \xi})^{3} \xi^{2}} \\
& +\frac{0.4805 r}{(1+\sqrt{r / \xi})^{2} \sqrt{r / \xi} \xi^{2}}-\frac{0.961}{(1+\sqrt{r / \xi}) \xi} \\
& -\frac{0.0755}{\sqrt{r / \xi}}+\frac{4.5585 \sqrt{r / \xi}}{(1+\sqrt{r / \xi})^{2} \xi} \\
& \left.+\frac{3.58(r / \xi)^{3 / 2}}{(1+\sqrt{r / \xi})^{4} \xi}+\frac{1-v}{\pi \xi} \frac{1}{r / \xi-1}\right]
\end{aligned}
$$

\section{RESULTS}

Figure 2 shows the interfacial contact pressure predicted for the three example cases. The coordinate axis has been normalized with respect to the distance to the violation point, $r_{\mathrm{c}}^{\mathrm{o}}$, and it may be seen that the actual closure length is shorter than the value implied by the bilateral solution. The difference increases with increasing value of $\alpha$. When the observation point is very close to the contact edge, it can be seen that the interfacial contact pressure is

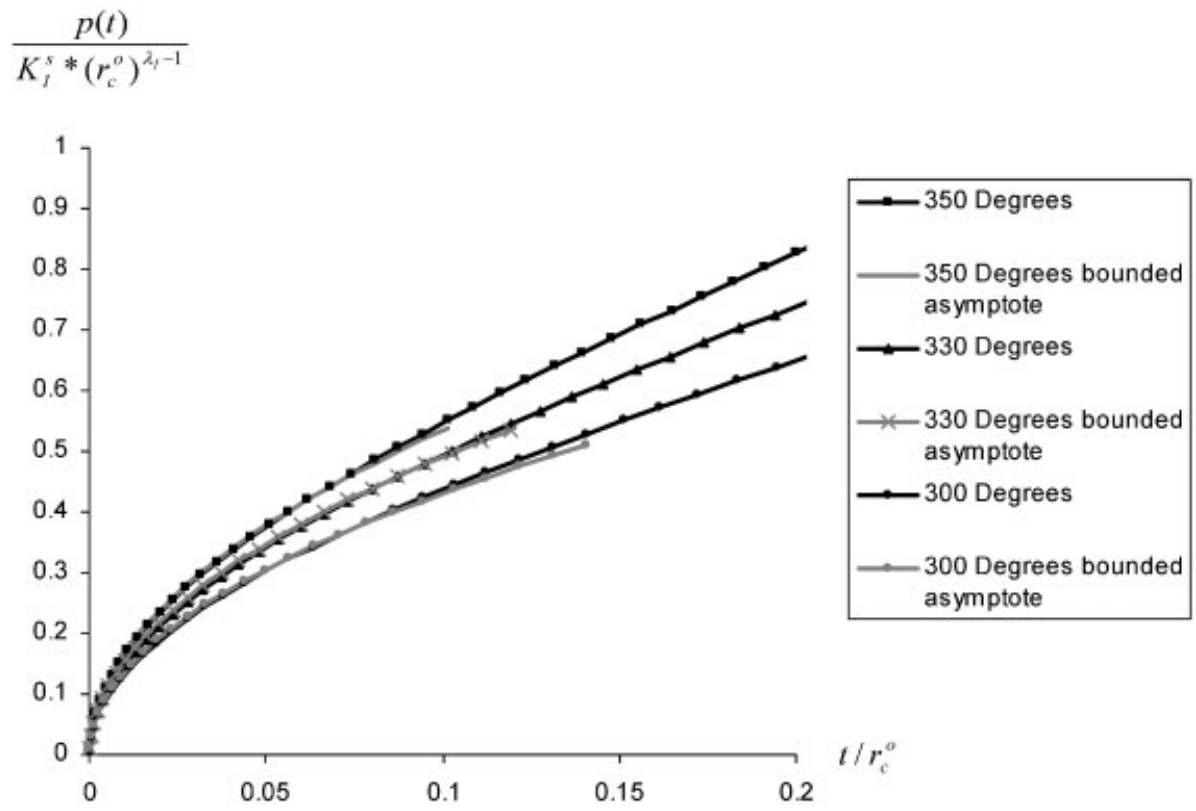

Fig. 3 A comparison of the true contact pressure near the point of separation and that implied by the bounded asymptote 
bounded and a connection between its value and the unilateral (open notch) stress distribution may be found. If $t$ is a coordinate measured inwards from the contact edge, the local contact pressure has the form

$$
p(t)=K_{\mathrm{I}}^{\mathrm{B}} t^{1 / 2}
$$

and the value of the multiplier may be found by interpolation of the form of the contact pressure (equation (9)) to the edges of the contact interval. Thus,

$$
K_{\mathrm{I}}^{\mathrm{B}}=C * \frac{K_{\mathrm{I}}^{\mathrm{s}}}{\left(r_{\mathrm{c}}^{\mathrm{o}}\right)^{3 / 2-\lambda_{\mathrm{I}}}}
$$

and the values of $\lambda_{\mathrm{I}}$ and $C$ for the three cases studied, together with a further intermediate angle, are given in Table 1. Figure 3 provides a close-up view of the true contact pressure adjacent to the edge of the contact region, and the contact pressure distribution implied by the asymptote.

\section{CONCLUSION}

When a remote compressive load is applied to a plate containing a sharp notch, closure will occur at the root. This phenomenon is normally neglected, because the solution is taken to be 'bilateral'. The implications of this assumption were examined in an earlier paper, simply by looking for violations in the displacement field, which implied interpenetration of material when the notch is compressed. The true closure length and interfacial contact pressure are found in this paper. The contact pressure between the notch faces is very large at the root but falls smoothly to zero in a square root fashion at the point of separation. The relationship between the bounded stress intensity factor $K_{\mathrm{I}}^{\mathrm{B}}$ (which characterizes the contact edge solution) and the generalized stress intensity factor $K_{\mathrm{I}}^{\mathrm{s}}$ (which relates to the crack root) is found. The true contact length is very close to that implied by interpenetration when the internal notch angle is not 'very large' (less than $300^{\circ}$ ), but the difference becomes much more marked when the notch becomes crack like in character.

\section{REFERENCES}

1 Churchman, C. M., Dini, D., and Hills, D. A. Closure at the root of a sharp notch. Int. J. Mech. Sci., 2006, 48, 1063-1071.

2 Qiu, H., Dini, D., and Hills, D. A. The influence function for self-equilibrating forces on a semiinfinite wedge. J. Strain Analysis, 2007, 42, 351-360.

3 Williams, M. L. Stress singularities resulting from various boundary conditions in angular plates in extension. Trans. ASME, J. Appl. Mechanics, 1952, 19, 526-528. 